\title{
Rare tumor of the ovary: Primitive choriocarcinoma*
}

\author{
Sanâa Errarhay ${ }^{1 \#}$, Najia Hmidani ${ }^{1}$, Samia Mahmoud ${ }^{1}$, Hind El Fatemi ${ }^{2}$, Hanane Saadi ${ }^{1}$, \\ Omar EI Mesbahi ${ }^{3}$, Nadia Squalli ${ }^{4}$, Chahrazed Bouchikhi ${ }^{1}$, Amarti Afaf ${ }^{2}$, Abdelaziz Banani ${ }^{1}$
}

\author{
${ }^{1}$ Department of Gynecology and Obstetrics, University Hospital of Fez, Fez, Morocco \\ ${ }^{2}$ Department of Pathology, University Hospital of Fez, Fez, Morocco \\ ${ }^{3}$ Department of Oncology, University Hospital of Fez, Fez, Morocco \\ ${ }^{4}$ Department of Radiology, University Hospital of Fez, Fez, Morocco \\ Email: "suine_err@yahoo.fr
}

Received 21 April 2013; revised 23 May 2013; accepted 1 June 2013

Copyright (c) 2013 Sanâa Errarhay et al. This is an open access article distributed under the Creative Commons Attribution License, which permits unrestricted use, distribution, and reproduction in any medium, provided the original work is properly cited.

\begin{abstract}
We report the case of a patient of 30 years with primary choriocarcinoma of the ovary. This patient referred for abdomino-pelvic mass suspected clinically and sonographically. Exploratory laparotomy was performed with the presence of two latero-uterine mass adherents to the highly vascularised uterus and difficult to resects deep plane with a mass left hepatic bleeding. A biopsy of both masses was performed and histological examination with immunehistochemical study returned for a primary ovarian non-gestational choriocarcinoma and the patient received 4 cycles of chemotherapy after hepatic artery embolization with good clinical, biological and radiological evaluation.
\end{abstract}

Keywords: Germinal Ovarian Tumour; Ovarian Choriocarcinoma; Chemotherapy

\section{INTRODUCTION}

The germinal tumors of the ovary are rare. They represent $1 \%$ of all the ovarian tumors but are aggressive. They affect preferentially the young women and are generally curable if they are prematurely diagnosed and treated with a rate of global survival in $85 \%$ in five years. The choriocarcinoma of the ovary can be gestationnal or not gestationnal, the latter exceptional is frequently associated with other germinal tumors as the dysgerminome, the embryonic carcinoma and the teratoma. We report a case of this unusual tumor with a review of the literature.

\footnotetext{
"Conflicts of interests: The authors declare to have no conflicts of interests in connection with this article.

Authors' contributions: E.S, H.N, M.S are the main authors who contributed to the writing of this article. E.H, S.H, M.O and S.N analyzed and interpreted the data of the patient and the review of the literature. A.A, B.C and B.A read and corrected the article.

"Corresponding author.
}

\section{OBSERVATION}

A 30-year-old patient, divorced and having benefited from an appendicectomy, an admitted person for coverage of an abdomino-pelvic mass with inanimate syndrome. The clinical examination finds a hard and painful abdomino-pelvic mass arriving up to the navel. The pelvic echography objectifies a retro-uterine tissular image making $10 \mathrm{~cm}$ vascularised in the Doppler. The abdomino-pelvic scanner shows the presence of two voluminous heterogeneous pelvic tumoral masses of $16 \mathrm{~cm}$ to the right and $14 \mathrm{~cm}$ to the left (Figure 1) with a left heterogeneous hepatic lesion of secondary rate (Figure 2). An exploratory laparotomy was realized with in the exploration presence of a hemo-peritoneum of low abundance and a peritoneal carcinosis with two latero-uterine masses at the expense of ovaries which seem very vascularised and sticky to the womb and to the deep framework difficult to resect with a left hepatic bleeding mass. A biopsy of both masses was realized and the anatomopathological study with the immuno-histochimical study returned in favour of an ovarian choriocarcinoma (Figure 3). A dosage of the rate of $\beta$ realized HCG plasma returned to $200 \mathrm{UI} / \mathrm{ml}$.

The patient benefited from an embolisation of the left hepatic artery. The post-operative consequences are simple. The patient presenting straightaway a vast pathology and requiring a treatment adjuvating of poly type chemotherapy. Chemotherapy with Bleomycine, Etoposide and Cisplatine is established. The patient receives 4 cures. After these 4 cures, she presents no more symptomatologies, the abdomino-pelvis scanner is normal, $\beta$ HCG is in $4 \mathrm{UI} / \mathrm{L}$ in 1 year. The patient is in clinical and biological and radiological complete remission.

\section{DISCUSSION}

The ovarian choriocarcinoma is a part of malignant germinal 


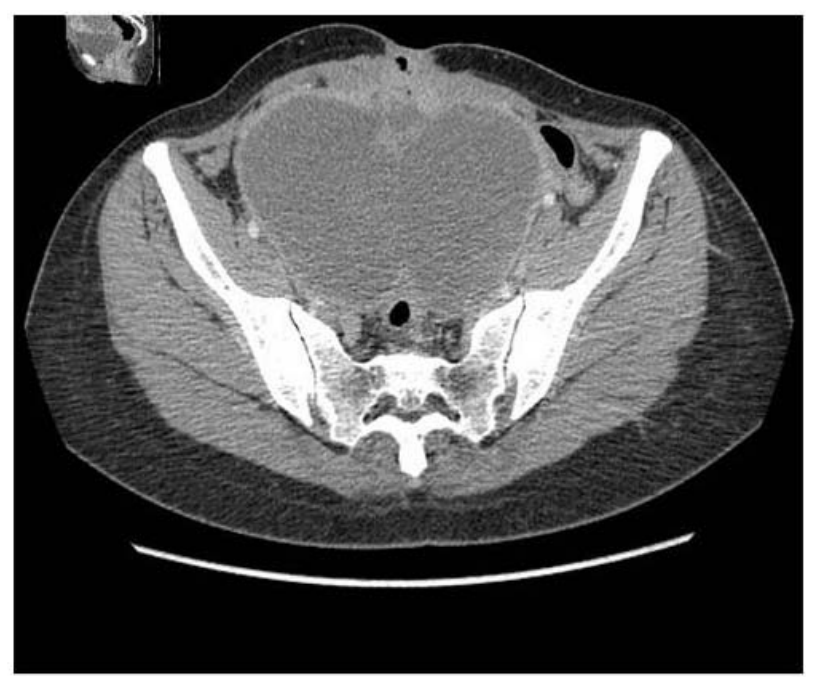

Figure 1. Computed tomographic examination of the abdomen and pelvis with intra venous-material: Presence of two voluminous pelvic, well limited masses, massively liquefied in fleshy enhanced components in periphery. The reconstructions in the coronal and sagittal plans allow localising them better on both sides of the womb.

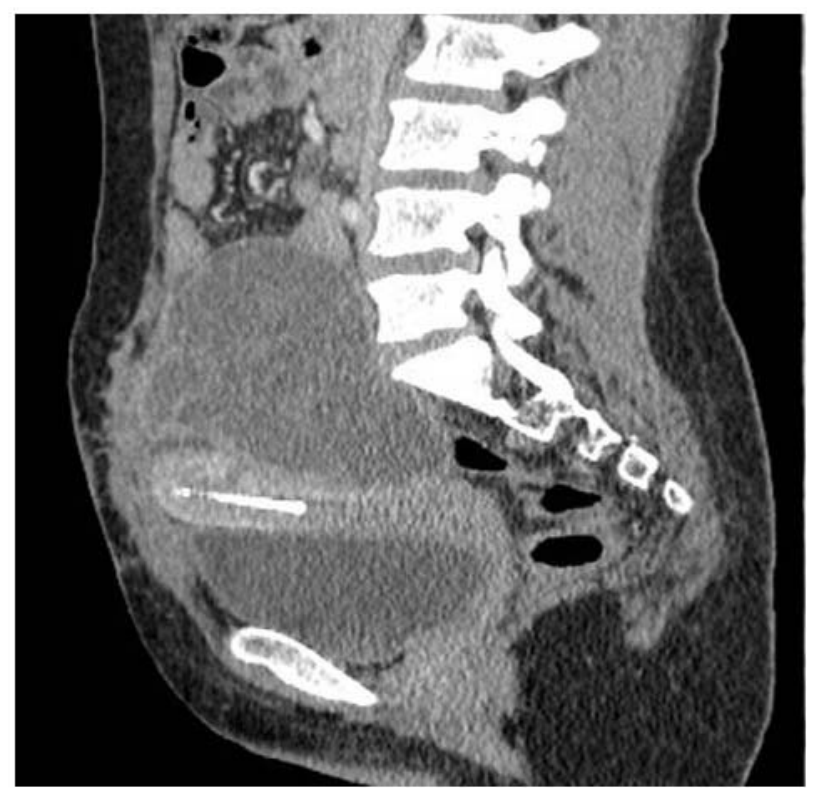

Figure 2. Computed tomographic examination of the abdomen and pelvis in reconstructions with intra venous-material: Voluminous hepatic mass occupying all the left liver, widely liquefied, enhanced in a heterogeneous way at the level of her tissular contingent, responsible for an overbooking of the dome diaphragmatic left and expulsion of the stomach downward.

tumors. They are defined by the presence of malignant cells, both cyto and syncitiotrophoblastic, closely intricate and without associated villositary formation [1,2] whose origin is either gestational (CCG) or non gestational (CCNG).

The primitive choriocarcinoma of the ovary is of very

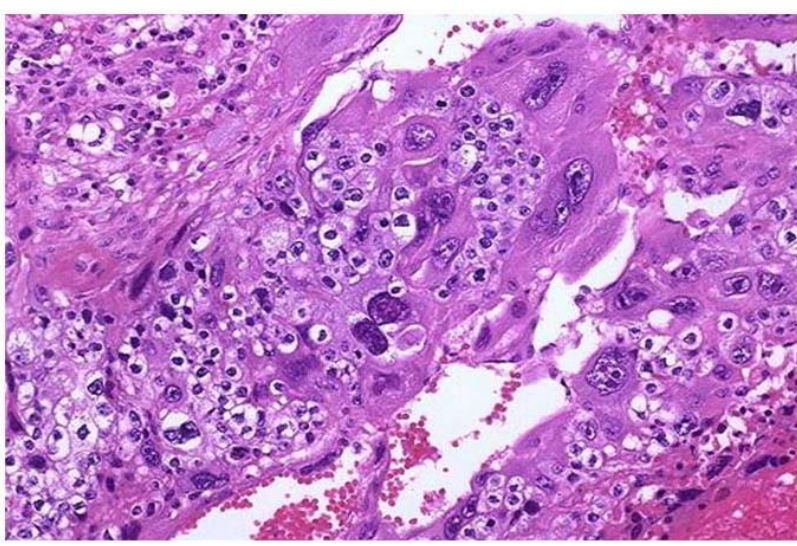

Figure 3. Optical microscopy: Incomplete spaces lined with syncitiotrophoblaste out of cytotrophoblaste and of intermediate cells.

rare diagnosis, described only in some cases in the literature, mostly at very prepubertal or virgin patients $[3,4]$. However, the diagnosis of primitive choriocarcinoma becomes extremely difficult in genital period of activity and most of the analyzed clinical cases are concerned with only very strong suspicions. The only possibility to distinguish with certainty the gestational choriocarcinoma from the primitive choriocarcinoma of the ovary consists of the analysis of the DNA on the operating room. The detection of the paternal antigens in the tumor reveals the gestational origin. However, these techniques are not systematically realized in current practice, as it was the case of our observation.

The treatment of the germinal tumors of the ovary is medico-surgical and depends on the histological type. The surgical stadification (total hysterectomy, bilateral annexectomy ommentectomy, ganglionic cleaning and peritoneal biopsies) must be realized to determine the forecast.

According to the histological results, adjuvating chemotherapy will be associated. For certain authors, the distinction between CCG and CCNG does not in any way seem essential because the treatment would depend essentially on the stage of the tumor, without consideration of its gestational or non gestational character $[5,6]$. For other authors, to differentiate the gestational and non gestational forms allows to propose better adapted treatments such as the methotrexate, particularly effective and tolerated well in the non metastatic gestational choriocarcinoma. On the other hand, non gestational tumors react less well to the methotrexate and require a polychemotherapy.

The forecast of choriocarcinoma is difficult to establish in the long term because of their rarity, in particular in case of CCNG. For the latter, the association surgery and adjuvating poly-chemotherapy seems to be a better treatment. The study of Goswami in $2001[4,7,8]$ about 
29 cases of CCNG, the rate of survival in 2 years was $81 \%$ with the patients having received an adjuvating chemotherapy against 28\% with the patients having not had this type of treatment. As for the CCG, their forecast seems better notably because of their sensibility to the methotrexate. These tumors are generally curable if they are prematurely diagnosed and treated with a rate of survival of $85 \%$ in five years.

The supervision of the ovarian CC rests on the clinical, ultrasound, CT scan as well as biological examination with the dosage of the HCG and its beta free fraction because it is about a highly specific and sensitive marker.

\section{CONCLUSION}

The distinction between gestational and non gestational choriocarcinoma is extremely difficult in genital period of activity. However, treatments used for the germinal tumors seem effective. The generalization of the genetic analyses of choriocarcinoma would allow bringing precision to optimize at best the coverage of these tumors in terms of survival and tolerance.

\section{REFERENCES}

[1] Norris, J.H. and O'connor, M.D. (1992) Pathology of malignant germ cell tumors of the ovary. In Coppleson, M., Ed., Gynaecologic Oncology, Longman Group (FE) Ltd., Hong Kong.

[2] Vance, R.P. and Geisinger, K.R. (1985) Pure non-gesta- tional choriocarcinoma of the ovary: Report of case. Cancer, 56, 2321-2325. doi:10.1002/1097-0142(19851101)56:9<2321::AID-CNC R2820560931>3.0.CO;2-Q

[3] Simsek, T., Track, B., Karaveli, M., Uner, M. and Sonmez, C. (1998) Primary pure choriocarcinoma of the ovary in reproductive ages: A case report. European Journal of Gynaecological Oncology, 19, 284-286.

[4] Goswami, D., Sharma, K., Zutshi, V., Tempe, A. and Nigam, S. (2001) Non gestational pure ovarian choriocarcinoma withcontrolateral teratoma. Gynecologic Oncology, 80, 262-266. doi:10.1006/gyno.2000.6043

[5] Jacobs, A.J., Newland, J.R. and Green, R.K. (1982) Pure choriocarcinoma of the ovary. Obstetrical \& Gynecological Survey, 37, 603-609. doi:10.1097/00006254-198210000-00001

[6] Benedet, J.L., Bender, H., Jones 3rd, H., Ngan, H.Y. and Pecorelli, S. (2000) FIGO staging classifications and clinical practice guidelines in the management of gynecologic cancers. FIGO Committee on Gynecologic Oncology. International Journal of Gynecology \& Obstetrics, 70, 209-262.

[7] Jain, T., Van Kessel, K., Reed, S. and Paley, P. (2000) Leydig cell tumor, mature teratoma, and non gestational choriocarcinoma in a single ovary. Obstetrics \& Gynecology, 95, 1031. doi:10.1016/S0029-7844(00)00876-0

[8] Jondle, D.M., Shahin, M.S., Sorosky, J. and Benda, J.A. (2002) Ovarian mixed germ cell tumor with predominance of polyembryoma: A case report with literature review. International Journal of Gynecological Pathology, 21, 78-81. doi:10.1097/00004347-200201000-00015 